\title{
Fuzzy Based Adaptive Controller for on - Chip Communication
}

\author{
M. Jasmin ${ }^{*}$, T. Vigneswaran ${ }^{2}$ \\ ${ }^{1}$ Research Scholar, Department of ECE, BIHER, Chennai, India. \\ ${ }^{2}$ Professor, School of Electronics, VIT, Chennai Campus, India.
}

\begin{abstract}
Occurrence of bit error is more when communication takes place in System on chip environment. By employing proper error detection and correction codes the bit error rate can be considerably reduced in On-chip communication. As System on chip involves heterogeneous system the efficiency of communication is improved when reconfigurable multiple coding schemes are preferred. Depending upon the requirements for various subsystem the correct code has to be selected. Due to the variations in input demands based on various subsystems the proper selection of codes become fuzzy in nature. In this paper Fuzzy Controller is designed to select the correct coding scheme. Inputs are given to the fuzzy controller based on the application demand of the user. The input parameters are minimum bit error rate, computational complexity and correlation level of the input data. Fuzzy Controller employs three membership functions and 27 rules to select the appropriate coding scheme. The selected coding scheme should be communicated at the proper time to the decoder. To enable the decoding process selected coding scheme is communicated effectively by using less overhead frame format. To verify the functionality of fuzzy controller random input data sets are used for testing.
\end{abstract}

Keywords: Error control, reconfigurable, SoC, communication, fuzzy controller.

\section{Introduction}

Network-on-chip (NoC) is endorsed in communication for its scalability and recyclability. Jammed regions can be defended by framing a novel routing set of rules by employing fuzzy- based routing choice.

The effective path for routing is [1] achieved through fuzzy controller and it also takes into account the power consumption .The availability of unused slots in input buffer of the neighbour cores is also considered.

The adapted methodology can effectively manage power sharing and low hardware overhead is achieved.

Network on the basis of On-chip interconnection is the better approach for managing the restricting aspect in System-on-chip (SoC) [2].

Network on Chip serves as a successful technique to the prevailing architecture used in interconnection as they satisfy the following traits; (i) power efficiency and reliability; (ii) scalability of bandwidth when in comparison to conventional bus architecture; (iii) reusability; (iv) allotted routing set of rules [3, 4]. Noc contributes a major role in transferring data between IP cores that are located in Soc.

In the previous models, the link that exists in the packet between the source and the destination is decided by these two nodes itself .The current traffic scenario is not taken into account which in turn affects the routing process.

This in turn degrades the throughput when the rate of packet injection increases. The routing based on adaptive method is done based on the status of network as the packet is traversing to reach its delivery point.

Algorithms based on adaptive routing methodology relies mainly on the network's status.

The performance is achieved at a higher rate and also power dissipation is lowered when Hybrid wireless Network-on-Chip
(NoC) is used[6] but, creation of on-chip wireless links lowers the general reliability of the communication.

Developing integrated systems with many cores is very advantageous and at the same time it put forth many challenges. As multiple cores are integrated density of the device is increased considerably but the underlying challenge encounters on reducing the power dissipation to minimum.

As said through (ITRS) in 2007, the contribution of interconnects to dissipation of power can escalate from $51 \%$ to up to $80 \%$ during the next five years. This manifestly indicates the challenges that has to be met in future related to conventional scaling of metallic interrelates. To attain an upgraded performance few appreciably in contrast to interconnect technology are being presently explored together with 3D integration [7], photonic interconnects [8] and multi-band RF [9] or wireless interconnects [10].

These new technologies had been proposed to enable designs involving multiple cores to improve the speed and performance. But these replacement interconnect technologies are in need to overcome critical demanding situations affecting to reliability. Error Control Coding (ECC) serves as a possible approach to improve the link dependability in On- chip Communication[11]. It has already been validated that ECCs specific design methods improve reliability in addition to reduced energy dissipation.

\section{System Model}

The system model is discussed with two block diagram 1) Fuzzy based coding scheme selection 2) The typical communication system using the fuzzy based coding scheme.

Figure 1 shows the fuzzy controller based coding scheme. 


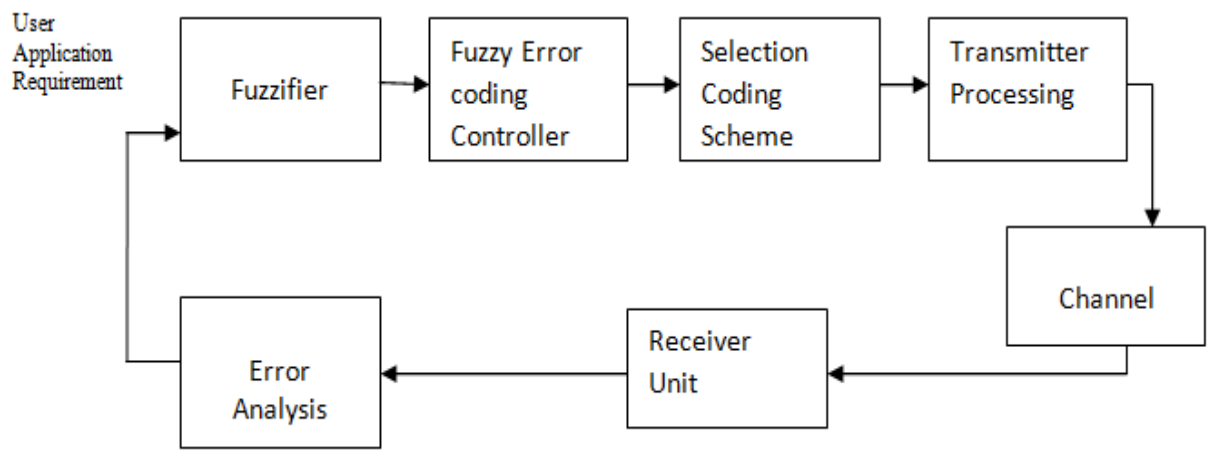

Fig. 1: Fuzzy based coding selection

The user application requirement information is sent to the fuzzier. The fuzzy parameter is given to the fuzzy error coding controller to the selection of optimal coding scheme by using fuzzy rules. The selected coding scheme process the input information bits and it is packetized in transmitter processing. The output is given to the channel. The receiver receives the packets, extracts the coded bits and select the coding scheme .based on the coding scheme information on the packet. The data bits are decoded by using the appropriate decoder with correct parameters. Error rate will be analysed in error analysis block then this error information is given to the fuzzier.

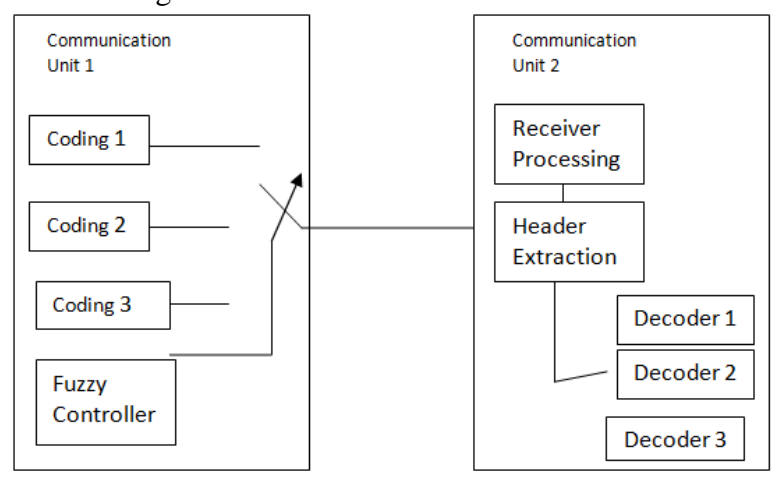

Fig. 2: Communication System

The communication system consists of two units namely Communication Unit 1 and Communication Unit2 .Communication unit 1 consists of many coding technique namely Coding 1, Coding 2, Coding 3, and Fuzzy Controller. Here Coding scheme 1 has to handle more error and the complex computation. Coding scheme 2 is used for moderate error handling with moderate complexity. Coding Scheme 3 act as less computational scheme with less error handling.

The fuzzy controller selects any one of the technique. Then the output is given to the communication unit 2. The communication unit 2 consists of receiver processing, header extractor and three decoders. The packets are received by the receiver processing then they are extracted by the header extractor and decoded by the decoder.

8bits 4 bits 4 bits 4 bits 4 bits 8 bits

\begin{tabular}{|c|c|c|c|c|c|}
\hline SD & SA & DA & CS & $\begin{array}{c}\text { PAY } \\
\text { LOAD }\end{array}$ & ED \\
\hline 8bits & 4 bits 4 bits 4 bits 4 bits 8 bits \\
\hline SD & SA & DA & $\begin{array}{c}\text { OPT. } \\
\text { PAY }\end{array}$ & DATA & ED \\
\hline
\end{tabular}

Fig. 3: Frame format

Where

SD -Start Delimentry

SA-Source Address

DA-Designation Address

\section{Cs- Code Selection}

ED-End Delimentry

Figure 3 shows the frame format used by the transmitter processing .Upper one is used to communicate the selected coding scheme using CS field. Lower one is used to communicate the operating parameters if there is any change from the previously sent parameters. If the operating parameter remains same the second format is not used. After receiving the changed coding scheme receiver will wait for the second format as next packet If it does not receive it then it is assumed that there is no change in the parameters. This implicit message passing reduces the overhead.

\section{Result and Discussion}

A fuzzy controller is designed to make the decision of selection of appropriate error control coding scheme based on the requirement of application and error channel condition and feedback from the receiving subsystem. Three input variables are introduced to capture 1) error control capability requirement of application (Error level) 2.computatational complexity of the scheme 3.correleation level of input data set. The three input variables values are mapped into fuzzy value by three triangle membership functions with a range value of zero to ten.

Table 1: Input Variables and Membership Function

\begin{tabular}{|l|l|l||}
\hline Name & Range & Number of membership functions \\
\hline Error_level & $0->10$ & 3 \\
\hline Encoding_comp & $0->10$ & 3 \\
\hline Correlation_lev & $0->10$ & 3 \\
\hline
\end{tabular}

Table 2: Output Variable and Output Membership Function

\begin{tabular}{|l|l|l|}
\hline Name & Range & Number of membership functions \\
\hline Selected_code & $0 \rightarrow 10$ & 3 \\
\hline
\end{tabular}

One output variable named "selected code" is used to capture the final selected code from the fuzzy controller. This variable output value is de mapped into crisp value by three triangle output membership function. Table 1 and table 2 gives the range of input and output variable and membership function as a sample, error_level input variable membership value split up details are given in table 3 .

Table 3: Error_Level Variable Membership Function

\begin{tabular}{|c|c|c|}
\hline Membership function & Shape & Points \\
\hline low_error & Triangle & $0 ; 1.75 ; 3$ \\
\hline med_error & Triangle & $2 ; 5 ; 7$ \\
\hline high_error & Triangle & $5 ; 8 ; 10$ \\
\hline
\end{tabular}



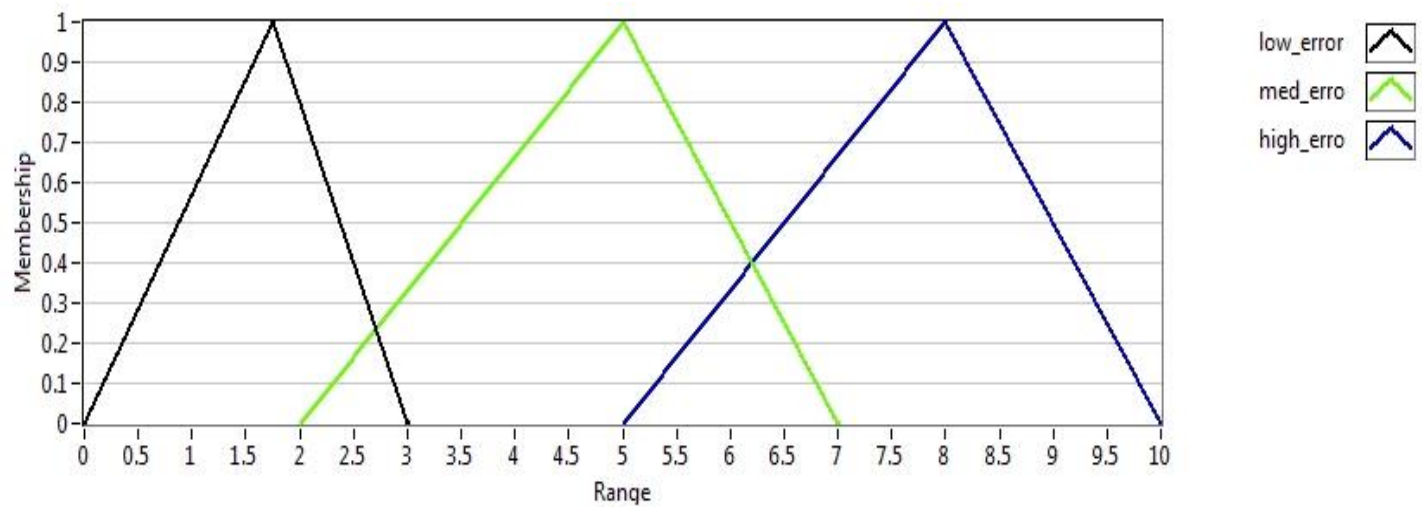

Fig. 4: Error level input membership function

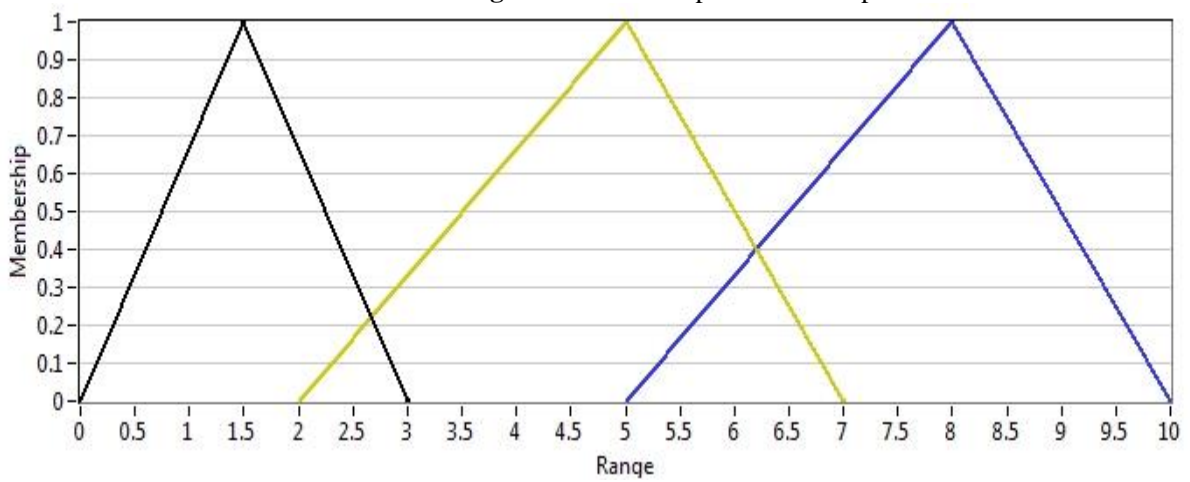

low_com
mid_com
high_co

Fig. 5: Computational complexity input membership function

Figure 4 to 6 gives the screen shot of the designed membership function for the three input variables. Figure 7 gives the screen shot of the output membership function.
For all membership function the range of values are fixed as zero to ten and the low level values for all the membership function are fixed as zero to 3 .mid-range values are taken from 2 to 7 and the high range values are taken as 5 to 10 .

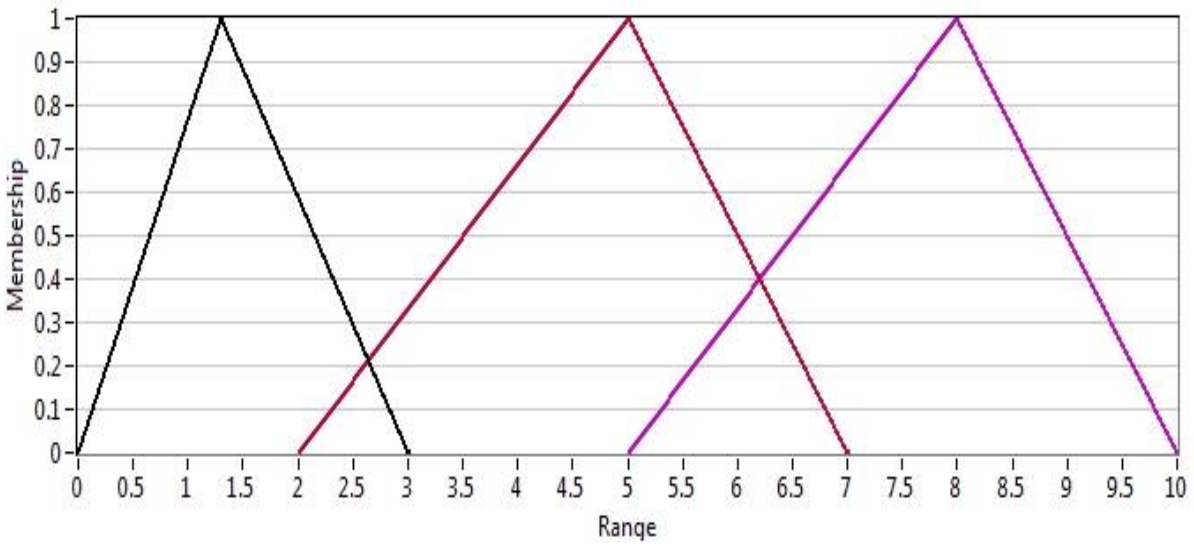

Low_corr med_corr high_corr

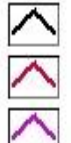

Fig. 6: Error handling level input membership function

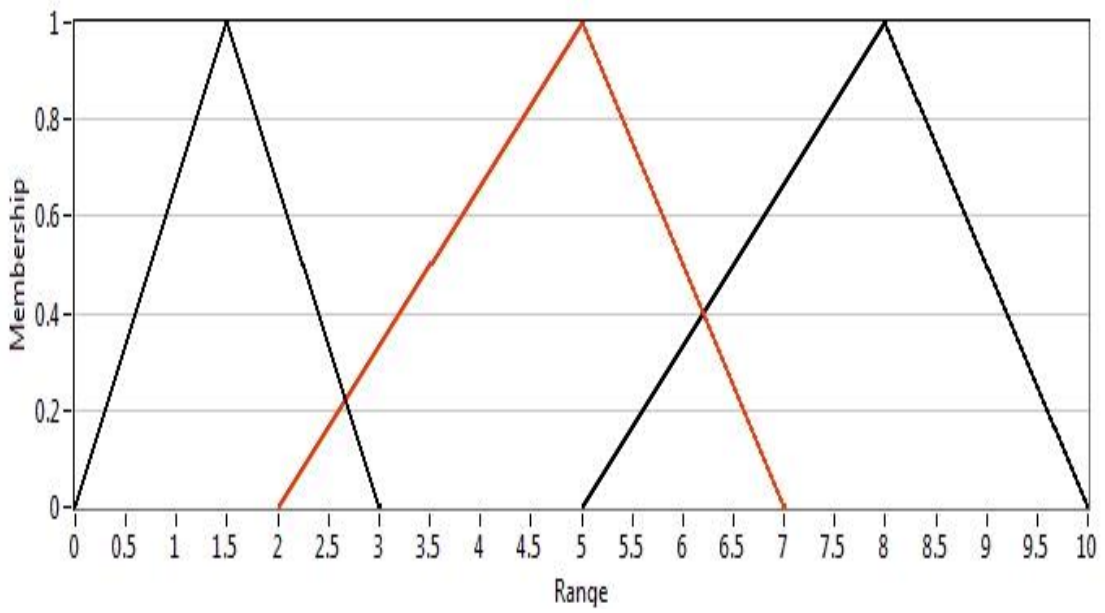

codel

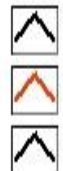

Fig. 7: Output membership function 
To select appropriate error coding scheme based on the three input variables 27 fuzzy rules are formulated [14].The 27 rules are used by the fuzzy controller and based on executed rule appropriate coding scheme is selected if more than one rule is executed under given input values, more weight-age value having code selected as a final selected code.[15]

Table 4: Fuzzy Inference Rules

1. IF 'Error level' IS 'low error' AND 'encoding comp' IS 'low_com' AND 'correlation_lev' IS 'Low_corr' THEN 'selected_code' IS 'code3'

connective: AND (Minimum) ; implication: Minimum ; degree of support: 1.00

2. IF 'Error_level' IS 'low_error' AND 'encoding_comp' IS 'low_com' AND 'correlation_lev' IS 'med_corr' THEN 'selected_code' IS 'code3'

connective: AND (Minimum) ; implication: Minimum ; degree of support: 1.00

3. IF 'Error_level' IS 'low_error' AND 'encoding_comp' IS 'low_com' AND 'correlation_lev' IS 'high_corr' THEN 'selected_code' IS 'code3'

connective: AND (Minimum) ; implication: Minimum ; degree of support: 1.00

4. IF 'Error_level' IS 'low_error' AND 'encoding_comp' IS 'mid_com' AND 'correlation_lev' IS 'Low_corr' THEN 'selected_code' IS 'code3'

connective: AND (Minimum) ; implication: Minimum ; degree of support: 1.00

5. IF 'Error_level' IS 'low_error' AND 'encoding_comp' IS 'mid_com' AND 'correlation_lev' IS 'med_corr' THEN 'selected_code' IS 'code2'

connective: AND (Minimum) ; implication: Minimum ; degree of support: 1.00

6. IF 'Error_level' IS 'low_error' AND 'encoding_comp' IS 'mid_com' AND 'correlation_lev' IS 'high_corr' THEN 'selected_code' IS 'code2'

connective: AND (Minimum) ; implication: Minimum ; degree of support: 1.00

7. IF 'Error_level' IS 'low_error' AND 'encoding_comp' IS 'high_com' AND 'correlation_lev' IS 'Low_corr' THEN 'selected_code' IS 'code1'

connective: AND (Minimum) ; implication: Minimum ; degree of support: 1.00

8. IF 'Error_level' IS 'low_error' AND 'encoding_comp' IS 'high_com' AND 'correlation_lev' IS 'med_corr' THEN 'selected_code' IS 'code2' connective: AND (Minimum) ; implication: Minimum ; degree of support: 1.00

9. IF 'Error_level' IS 'low_error' AND 'encoding_comp' IS 'high_com' AND 'correlation_lev' IS 'high_corr' THEN 'selected_code' IS 'code1'

connective: AND (Minimum) ; implication: Minimum ; degree of support: 1.00

10. IF 'Error_level' IS 'med_error' AND 'encoding_comp' IS 'low_com' AND 'correlation_lev' IS 'Low_corr' THEN 'selected_code' IS 'code2'

connective: AND (Minimum) ; implication: Minimum ; degree of support: 1.00

11. IF 'Error_level' IS 'med_error' AND 'encoding_comp' IS 'low_com' AND 'correlation_lev' IS 'med_corr' THEN 'selected_code' IS 'code3'

connective: AND (Minimum) ; implication: Minimum ; degree of support: 1.00

12. IF 'Error_level' IS 'med_error' AND 'encoding_comp' IS 'low_com' AND 'correlation_lev' IS 'high_corr' THEN 'selected_code' IS 'code2'

connective: AND (Minimum) ; implication: Minimum ; degree of support: 1.00

13. IF 'Error_level' IS 'med_error' AND 'encoding_comp' IS 'mid_com' AND 'correlation_lev' IS 'Low_corr' THEN 'selected_code' IS 'code2'

connective: AND (Minimum) ; implication: Minimum ; degree of support: 1.00

14. IF 'Error_level' IS 'med_error' AND 'encoding_comp' IS 'mid_com' AND 'correlation_lev' IS 'med_corr' THEN 'selected_code' IS 'code2'

connective: AND (Minimum) ; implication: Minimum ; degree of support: 1.00

15. IF 'Error_level' IS 'med_error' AND 'encoding_comp' IS 'mid_com' AND 'correlation_lev' IS ' 'high_corr' THEN 'selected_code' IS 'code2' connective: AND (Minimum) ; implication: Minimum ; degree of support: 1.00

16. IF 'Error_level' IS 'med_error' AND 'encoding_comp' IS 'high_com' AND 'correlation_lev' IS 'Low_corr' THEN 'selected_code' IS 'code1'

connective: AND (Minimum) ; implication: Minimum ; degree of support: 1.00

17. IF 'Error_level' IS 'med_error' AND 'encoding_comp' IS 'high_com' AND 'correlation_lev' IS 'med_corr' THEN 'selected_code' IS 'code2'

connective: AND (Minimum) ; implication: Minimum ; degree of support: 1.00

18. IF 'Error_level' IS 'med_error' AND 'encoding_comp' IS 'high_com' AND 'correlation_lev' IS 'high_corr' THEN 'selected_code' IS 'code3'

connective: AND (Minimum) ; implication: Minimum ; degree of support: 1.00

19. IF 'Error_level' IS 'high_error' AND 'encoding_comp' IS 'low_com' AND 'correlation_lev' IS 'Low_corr' THEN 'selected_code' IS 'code2'

connective: AND (Minimum) ; implication: Minimum ; degree of support: 1.00

20. IF 'Error_level' IS 'high_error' AND 'encoding_comp' IS 'low_com' AND 'correlation_lev' IS 'med_corr' THEN 'selected_code' IS 'code2'

connective: AND (Minimum) ; implication: Minimum ; degree of support: 1.00

21. IF 'Error_level' IS 'high_error' AND 'encoding_comp' IS 'low_com' AND 'correlation_lev' IS 'high_corr' THEN 'selected_code' IS 'code2'

connective: AND (Minimum) ; implication: Minimum ; degree of support: 1.00

22. IF 'Error_level' IS 'high_error' AND 'encoding_comp' IS 'mid_com' AND 'correlation_lev' IS 
'Low_corr' THEN 'selected_code' IS 'code2'

connective: AND (Minimum) ; implication: Minimum ; degree of support: 1.00

23. IF 'Error_level' IS 'high_error' AND 'encoding_comp' IS 'mid_com' AND 'correlation_lev' IS 'med_corr' THEN 'selected_code' IS 'code2' connective: AND (Minimum) ; implication: Minimum ; degree of support: 1.00

24. IF 'Error_level' IS 'high_error' AND 'encoding_comp' IS 'mid_com' AND 'correlation_lev' IS 'high_corr' THEN 'selected_code' IS 'code2'

connective: AND (Minimum) ; implication: Minimum ; degree of support: 1.00

25. IF 'Error_level' IS 'high_error' AND 'encoding_comp' IS 'high_com' AND 'correlation_lev' IS 'Low_corr' THEN 'selected_code' IS 'code1'

connective: AND (Minimum) ; implication: Minimum ; degree of support: 1.00

26. IF 'Error_level' IS 'high_error' AND 'encoding_comp' IS 'high_com' AND 'correlation_lev' IS 'med_corr' THEN 'selected_code' IS 'code1'

connective: AND (Minimum) ; implication: Minimum ; degree of support: 1.00

27. IF 'Error_level' IS 'high_error' AND 'encoding_comp' IS 'high_com' AND 'correlation_lev' IS 'high_corr' THEN 'selected_code' IS 'code1' connective: AND (Minimum) ; implication: Minimum ; degree of support: 1.00

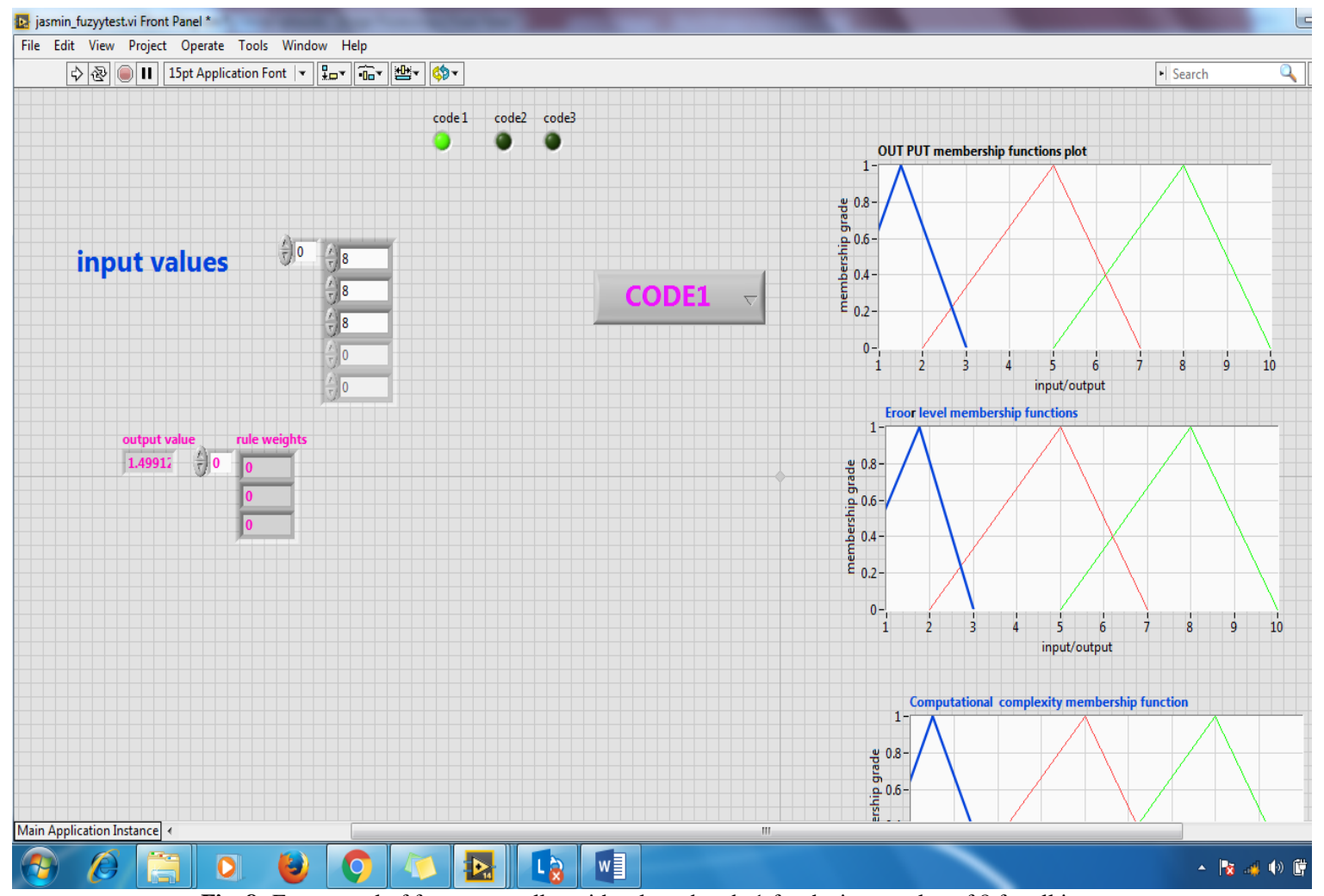

Fig. 8: Front panel of fuzzy controller with selected code 1 for the input value of 8 for all inputs

Figure 8 shows the screen shot of output of the fuzzy controller which selects code 1 because the output value is 1.4 .

\section{Conclusion}

Selecting appropriate coding scheme for the heterogeneous SOC is fuzzy in nature because different subsystem requires different computational complexity level of error control coding. Similarly, different application demands different error control capability for the successful deployment of the application . Error control capability of coding scheme can be extended by exploiting the correlation level, Computational Complexity and bit error rate of the input data sets. Handing all the above three elements to select best code is successfully implemented by means of a fuzzy controller and results are presented.

\section{References}

[1] Nastaran S \& Arash D, “A Fuzzy-Based Power-Aware Routing Algorithm for Network on Chip", 12th International Conference on Advanced Communication Technology, (2010), pp.1159-1163.

[2] Dally WJ \& Towles B, "Route Packets, Not Wires: On-Chip Interconnection Networks", IEEE Proceedings of the Design Automation Conference, Yokohama, Japan, (2001), pp.684-689.

[3] Ivanov A \& Micheli GD, "The Network -on-Chip Paradigm in Practice and Research", IEEE Design and Test of Computers, Vol.22, No.5, (2005), pp.399-403.

[4] Guerrier P \& Greiner A, "A generic architecture for on-chip packet-switched interconnections", Design, Automation and Test in Europe (DATE'00), (2000), pp.250-256.

[5] Acsia G, Catania V, Pales M \& Patti D, "A New Selection Strategy for on-Chip Networks", IEEE Embedded Systems for Real Time multimedia, Vol.22, No.5, (2008), pp.79-84.

[6] Amlan G, Partha P, Benjamin B \& Alireza N, "A Unified Error Control Coding Scheme to Enhance the Reliability of a Hybrid Wireless Network-on-Chip", IEEE International Symposium on Defect and Fault Tolerance in VLSI and Nanotechnology Systems (DFT), (2011), pp.277-285. 
[7] Pavlidis VF \& Friedman EG, "3-D Topologies for Networks-onChip", IEEE Transactions on Very Large Scale Integration (VLSI), Vol.15, No. 10, (2007), pp.1081-1090.

[8] Shacham A, "Photonic Network-on-Chip for Future Generations of Chip Multi-Processors", IEEE Transactions on Computers, Vol.57, No.9, (2008), pp.1246-1260.

[9] Chang MF, "CMP Network-on-Chip Overlaid With Multi-Band RF-Interconnect", IEEE International Symposium on High Performance Computer Architecture (HPCA), (2008), pp.191-202.

[10] Zhao D \& Wang Y, "SD-MAC: Design and Synthesis of a Hardware-Efficient Collision-Free QoS-Aware MAC Protocol for Wireless Network-on Chip", IEEE Transactions on Computers, Vol.57, No.9, (2008), pp.1230-1245.

[11] Sridhara SR \& Shanbhag NR, "Coding for System-on-Chip Networks: A Unified Framework", IEEE Transactions on Ver Large Scale Integration (TVLSI) Systems, Vol.13, No.6, (2005), pp.655-667.

[12] Ganguly A, "Crosstalk-Aware Channel Coding Schemes for Energy Efficient and Reliable NoC Interconnects", IEEE Transactions on VLSI (TVLSI), Vol.17, No.(11), (2009), pp.1626-1639.

[13] Phan, HP \& Tran, XT, "A fuzzy-logic based voltage-frequency controller for network-on-chip routers", IEEE 11th Conference on Ph.D. Research in Microelectronics and Electronics (PRIME), (2015), pp.192-195.

[14] Akhpanov, S. Sabitov, R. Shaykhadenov (2018). Criminal pre-trial proceedings in the Republic of Kazakhstan: Trend of the institutional transformations. Opción, Año 33. 107-125.

[15] D, Ibrayeva, Z Salkhanova, B Joldasbekova, Zh Bayanbayeva (2018). The specifics of the art autobiography genre. Opción, Año 33. 126-151. 